\title{
Synthesis and Antimicrobial Evaluation of Novel 4-phenylPyrrole-2-Carboxamides
}

\author{
Yogesh D Mane ${ }^{1 *}$, Santosh M Surwase ${ }^{2}$, Vishnu S Shinde ${ }^{2}$ and Bhimrao C Khade ${ }^{3}$ \\ ${ }^{1}$ BSS Arts, Science \& Commerce College, Makni, Dist-Osmanabad,M.S, India \\ ${ }^{2}$ Shri Chhatrapati Shivaji College, Omerga, Dist-Osmanabad,M.S, India \\ ${ }^{3}$ Dnyanopasak College, Parbhani, Dist- Parbhani, M.S, India \\ *Corresponding author: Yogesh D Mane, BSS Arts, Science \& Commerce College, Makni, Dist-Osmanabad,M.S. India
}

ARTICLE INFO

Received: 蔧 July 09, 2020

Published: 幽 August 17, 2020

Citation: Yogesh D Mane, Santosh M Surwase, Vishnu S Shinde and Bhimrao C Khade. Synthesis and Antimicrobial Evaluation of Novel 4-phenylPyrrole-2-Carboxamides. Biomed J Sci \& Tech Res 29(4)-2020. BJSTR. MS.ID.004824.

\section{ABSTRACT}

Ten novel 4-phenylpyrrole-2-carboxamide derivatives (5a-j) were synthesized and evaluated for their in vitro antibacterial activity. Among the tested compounds, the most effective were $5 \mathrm{c}$ and $5 \mathrm{e}$ with MIC value in the range of $6.05-6.25 \mu \mathrm{g} / \mathrm{mL}$ against Gram-negative bacterial strains. Further, the synthesized compounds have been screened for their in vitro antifungal activity. In the present study, novel 1-(4-chlorobenzyl)-4-phenyl-1H-pyrrole-2-carboxamides as antibacterial agents have been disclosed.

Keywords: Pyrrole-2-Carboxamide; Antibacterial and Antifungal Activity

\section{Introduction}

Drug resistance infection always creates a threat to healthcare. It becomes more acute due to the rapid development of resistance against conventional chemotherapy [1]. The necessity for more potent antimicrobial agents has become vital because of emerged resistance to the currently used antibiotics. In the last few decades, rapid scientific progress has been made in the treatment of infectious diseases. However, they still remain a serious and challenging health problem due to several factors which have led to the re-emergence of these diseases. Antibiotic resistance, population increase, international travel, migration, increase in the number of immune-suppressed patients, and climate change are some of the factors that play a significant role in the battle against infectious diseases [2-6]. In order to keep microorganisms resistance under control, careful use of existing antimicrobial drugs and the design of novel drugs with different modes of action e.g. linezolid [7-9] are required [10-13]. Pyrrole and its derivatives are ever present in nature. Pyrrole subunit has diverse applications in therapeutically active compounds including fungicides, antibiotics, anti-inflammatory drugs [14], antitumor agents [15], cholesterol reducing drugs [16] and many more. They are known to inhibit reverse transcriptase [Human immunodeficiency virus type 1 (HIV-1)] and cellular DNA polymerases protein kinases. Moreover, they are also a component of polymers [17], indigoid dyes and of larger aromatic rings [18]. In catalytic reactions, pyrroles are well utilized as catalyst for polymerization process [19], preservative [20], solvent for resin [21], corrosion inhibitor [22], terpenes and in metallurgical process [23].

One approach is to improve the activity of natural anti-microbial substances by synthesis of analog compounds of naturally produced organohalogens [24]. Our curiosity in halogenated pyrrole derivatives led to synthesis and antimicrobial evaluation of some analogues of pyoluteorin [25]. In continuation to this we found that bromopyrrole alkaloids; a family of marine alkaloids represents a fascinating example of the large variety of secondary metabolites formed by marine sponges. These compounds are involved in the sponge's defense mechanism against fishes. Also, several pharmacologically important bromopyrrole congeners have been previously described as having antihistaminic, antiserotonergic and antineoplastic activity. Furthermore, these natural products also possess antibacterial, antifungal and anitibiofilm activity 
[26-28]. Most of these compounds are defined by the signature of bromopyrrole carboxamide with oroidin as their prototype alkaloid reported for its antibiofilm activity [26]. Structure-activity relationship performed on synthetic library of oroidin derivatives indicated that $\mathrm{N}$-methylation of the pyrrole ring led to increased antibiofilm activity against medically relevant Gram-negative c-proteobacterium Pseudomonas aeruginosa, as indicated by the most active member of the library, Dihydrosventrin (DHS) [29].

The structural and therapeutic diversity coupled with the commercial viability of different types of small molecules has fascinated organic and medicinal chemists. The pyrrole-2carboxamide moiety is a pharmacophores found as a core skeleton in molecules with diverse biological activities such as insecticidal [30], antibiofilm [31], antibacterial [32], ATPase inhibitors of DNA gyrase [33], DNA binding, topoisomerase I inhibition [34], inducible nitric oxide synthase (nNOS and iNOS) inhibitors [35], antifungal [36], anticancer [37], JAK2 inhibitors [38], CB2 receptor antagonists [39], antitumor [40]. Representative biologically potent substituted pyrrole carboxamides are shown below (Figure 1). In continuation of research on pyrrole-2-carboxamide and to know the effect of lipophilic phenyl group on antibacterial potency of pyrrole-2-carboxamide [41] we report here the synthesis and antibacterial activity of novel 4-phenylpyrrole-2-carboxamide derived from corresponding carboxylic acid and different amines.

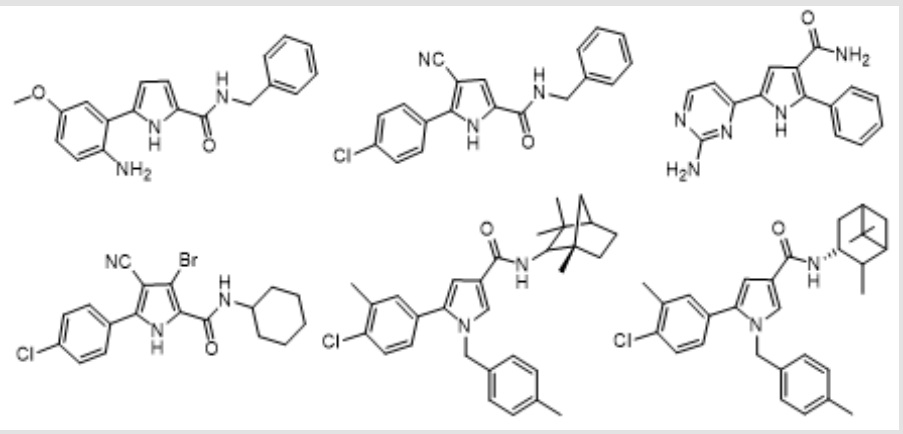

Figure 1: Reported pyrrole carboxamide based bioactive compounds.

\section{Results and Discussion}

\section{Chemistry}

The 4-phenylpyrrole-2-carboxamides (5a-j) have been synthesized following procedures sketched in the Scheme 1. The precursor ethyl 4-bromo-1-(4-chlorobenzyl)-1H-pyrrole-2carboxylate (2) has been prepared by N-benzylation of commercially available ethyl 4-bromo-1H-pyrrole-2-carboxylate (1) with 4-chlorobenzylchloride and Cs2CO3 in DMF. Ethyl 4-bromo-1-(4-
chlorobenzyl)-1H-pyrrole-2-carboxylate (2) on Suzuki coupling with phenylboronic acid in presence of $\mathrm{Pd}(\mathrm{dppf}) \mathrm{Cl} 2-\mathrm{CH} 2 \mathrm{Cl}$ and KOAc in 1,4-Dioxane afford ethyl 1-(4-chlorobenzyl)-4-phenyl-1Hpyrrole-2-carboxylate (3) in low yield. Ester 3 on hydrolysis with LiOH in THF, $\mathrm{H} 2 \mathrm{O}$ and EtOH system furnishes 1-(4-chlorobenzyl)4-phenyl-1H-pyrrole-2-carboxylic acid (4). Carboxylic acid (4) on coupling with appropriate substituted or non substituted aryl and cycloaliphatic amines using EDC $\cdot \mathrm{HCl}, \mathrm{HOBt}$ and DIPEA/TEA in DMF affords the target carboxamides ( $5 \mathrm{a}-\mathrm{j}$ ) in good to excellent yields.

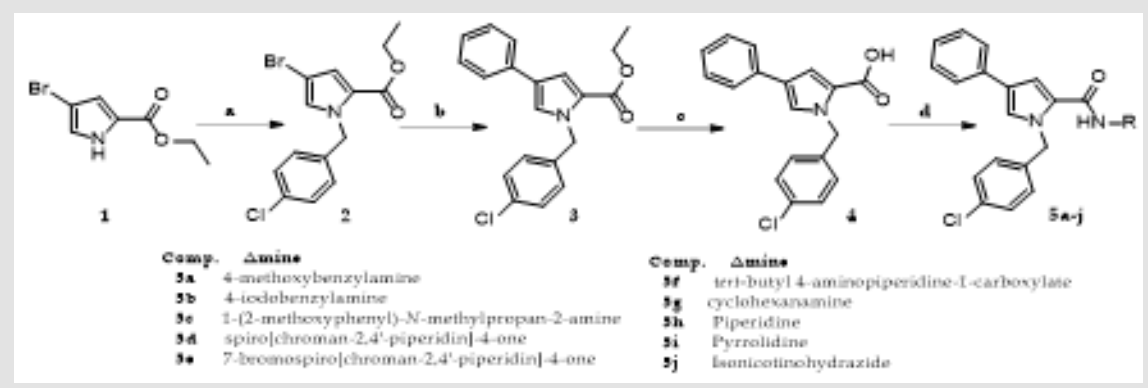

Scheme 1: Synthetic route of the 4-phenylpyrrole-2-carboxamides (5a-j).

Reagents and conditions: (a) 4-Chlorobenzyl Chloride, Cs2CO3, DMF, 60oC, 16 h; (b) Phenylboronic acid, Pd(dppf)Cl2$\mathrm{CH} 2 \mathrm{Cl}$ 2, K2CO3, CH3CN, H2O, 80-900C, 24 h; (c) LiOH, THF, H2O, EtOH, Stirr, rt, 3.5 h; (d) Amine, EDC.HCl, HOBt, DIPEA, DMF, 0oC to rt, $18-30 \mathrm{~h}$. 
The formation of compound $5 \mathrm{a}$ was confirmed by $1 \mathrm{H}$ NMR, 13C NMR and mass spectral analysis. In the $1 \mathrm{H}$ NMR spectrum of compound $5 \mathrm{a}, \mathrm{N}-\mathrm{H}$ proton of amide group observed at $\delta 9.3 \mathrm{ppm}$. The methylene groups attached to nitrogen of pyrrole ring and amide amide functionality showed singlet at $\delta 4.2$ and $4.8 \mathrm{ppm}$ respectively. In addition to this, the signal observed at $\delta 3.7 \mathrm{ppm}$ indicates the presence of the -OMe group on the benzene ring. In the 13C NMR spectrum of compound $5 \mathrm{a}$, the signal at $\delta 162.4 \mathrm{ppm}$ is due to amide carbonyl carbon. The signals at $\delta 42.8$ and $54.7 \mathrm{ppm}$ indicate the presence of methylene carbon attached to the nitrogen of the pyrrole ring and amide nitrogen atom. The presence of $[\mathrm{M}]+$ signal at m/z 430 in EIMS also confirms formation of compound 5a. Furthermore, formation of 4-phenylpyrrole-2-carboxamides (5b-j) was also confirmed by $1 \mathrm{H}$ NMR, 13C NMR and mass spectral analysis.

\section{Biological Evaluation}

Antibacterial Activity: The in vitro antimicrobial susceptibility of prepared compounds 5a-j against strains of pathogenic Gramnegative bacteria Klebsiella pneumoniae (ATCC 27736), Escherichia coli (ATCC 9637), Pseudomonas aeruginosa (ATCC BAA427) and Staphylococcus aureus (ATCC 25923) were evaluated by broth microdilution technique described by Clinical and Laboratory Standards Institute (CLSI), 2012 (Formerly NCCLS) [42]. The minimum inhibitory concentration (MIC, $\mu \mathrm{g} / \mathrm{mL}$ ) was defined as the lowest concentration of an antimicrobial agent that will inhibit the visible growth of microbe. Gentamicin and Ciprofloxacin were used as standard drugs for comparison of antibacterial activity. Dimethylsulfoxide (DMSO) was used as a solvent or negative control. To clarify any effect of DMSO on the antibacterial activity, separate studies were carried out with solutions alone of DMSO and these studies showed no activity against any microbial strains. The MIC of tested compounds was determined using the two fold serial dilution technique by assaying at 51.2, 25.6, 12.8, 6.4, 3.2, 1.6, 0.8, $0.4,0.2,0.1$ and $0.05 \mu \mathrm{g} / \mathrm{mL}$ concentrations along with standards at the same concentrations.

\section{Antifungal Activity}

The in vitro Antifungal Susceptibility (AFST) of prepared compounds 5a-j against strains of pathogenic fungi, for example, Candida albicans, Cryptococcus neoformans, Aspergillus fumigatus (all strains are patient's isolates) and Candida parapsilosis (ATCC 22019) were evaluated by broth microdilution technique described by Clinical and Laboratory Standards Institute (CLSI), $2012[43,44]$. Fluconazole and Oxiconazole were used as standard drugs for comparison of antifungal activity. DMSO was used as a solvent or negative control. To clarify any effect of DMSO on the antifungal activity, separate studies were carried out with solutions alone of DMSO and these studies showed no activity against any microbial strains. The MIC it is the lowest concentration of an antimicrobial agent that will inhibit the visible growth of microbe of tested compounds was determined using the two fold serial dilution technique by assaying at $64,32,16,8,4,2,1$ and $0.5 \mu \mathrm{g} / \mathrm{mL}$ concentrations along with standards at the same concentrations. The results of antibacterial activity of the tested compounds (5a-j) are shown in Table 1. The antibacterial activities were tested using reference standards Gentamicin and Ciprofloxacin. The biological evaluation clearly exhibits that two of the synthesized compounds show considerable antibacterial activity against clinical isolates of E.coli, P. aeruginosa or K. pneumoniae with MIC values in the range of 6.05-6.25 $\mu \mathrm{g} / \mathrm{mL}$. Compound 5c showed considerable antibacterial activity against E.coli, and P. aeruginosa with MIC values of $6.05 \mu \mathrm{g} /$ $\mathrm{mL}$. Further, compound 5e also showed considerable antibacterial activity against K. pneumoniae with MIC values of $6.25 \mu \mathrm{g} / \mathrm{mL}$. The results of antifungal activity of the tested compounds $5 \mathrm{a}-\mathrm{j}$ are presented in Tables 1 . The antifungal activities were tested using reference standards Fluconazole and Oxiconazole. Amongst series the only $5 \mathrm{a}$ and $5 \mathrm{c}$ was found to show moderate activity against the tested C. neoformans and A. fumigatus strains. Remaining derivatives does not show any significant antifungal activity against the tested strains.

Table 1: In vitro antimicrobial activity of compounds (5a-j) against pathogenic Gram-Negative bacteria \& fungi.

\begin{tabular}{|c|c|c|c|c|c|c|c|c|}
\hline \multirow{2}{*}{ Compd. } & \multicolumn{4}{|c|}{ MIC $(\mu \mathrm{g} / \mathrm{mL})$} & \multicolumn{4}{c|}{ MIC $(\boldsymbol{\mu g} / \mathrm{mL})$} \\
\cline { 2 - 9 } & $\begin{array}{c}\text { Klebsiella } \\
\text { pneumoniae }\end{array}$ & $\begin{array}{c}\text { Escherichia } \\
\text { coli. }\end{array}$ & P aeruginosa & $\begin{array}{c}\text { Salmonella } \\
\text { Typhi }\end{array}$ & $\begin{array}{c}\text { C. } \\
\text { albicans }\end{array}$ & $\begin{array}{c}\text { C. } \\
\text { neoformans }\end{array}$ & $\begin{array}{c}\text { A. } \\
\text { fumigatus }\end{array}$ & $\begin{array}{c}\text { C. } \\
\text { parapsilosis }\end{array}$ \\
\hline $5 \mathrm{a}$ & 50 & 25.5 & 12.05 & $>50$ & $>50$ & 25 & 50 & $>50$ \\
\hline $5 \mathrm{~b}$ & 12.5 & 25 & 12.25 & $>50$ & $>50$ & $>50$ & $>50$ & $>50$ \\
\hline $5 \mathrm{c}$ & 50 & 6.05 & 6.05 & $>50$ & $>50$ & 25 & 25 & $>50$ \\
\hline $5 \mathrm{~d}$ & 25.5 & 12.25 & 12.25 & $>50$ & $>50$ & $>50$ & $>50$ & $>50$ \\
\hline $5 \mathrm{e}$ & 6.25 & 12.5 & 50 & $>50$ & $>50$ & $>50$ & $>50$ & $>50$ \\
\hline $5 \mathrm{f}$ & 25.5 & 12.5 & 50 & $>50$ & $>50$ & $>50$ & $>50$ & $>50$ \\
\hline $5 \mathrm{~g}$ & 12.5 & 25.5 & 12.2 & 50 & $>50$ & $>50$ & $>50$ & $>50$ \\
\hline $5 \mathrm{~h}$ & 50 & 12.2 & 25.5 & 12.05 & $>50$ & $>50$ & $>50$ & $>50$ \\
\hline $5 \mathrm{i}$ & 25.5 & 12.05 & 12.5 & $>50$ & $>50$ & $>50$ & $>50$ & $>50$ \\
\hline
\end{tabular}




\begin{tabular}{|c|c|c|c|c|c|c|c|c|}
\hline $5 j$ & $>50$ & $>50$ & $>50$ & $>50$ & $>50$ & $>50$ & $>50$ & $>50$ \\
\hline $\begin{array}{c}\text { Gentamicin } \\
\text { (Fluconazole) }\end{array}$ & 0.25 & 1.25 & 3.025 & 1.65 & -0.5 & -1 & -2 & -1 \\
\hline $\begin{array}{l}\text { Ciprofloxacin } \\
\text { (Oxiconazole) }\end{array}$ & 0.5 & 1.05 & 1.25 & 3.01 & -0.03 & -1.4 & -2 & -0.01 \\
\hline
\end{tabular}

\section{Experimental}

\section{Material and Methods}

Reagents and solvents were purchased from commercial sources and used without further purification unless otherwise specified. Melting points were determined in open capillary tubes and are uncorrected. Formation of the compounds was checked by TLC on silica gel-G plates of $0.5 \mathrm{~mm}$ thickness and spots were located by iodine and UV light. All compounds were purified by recrystallization/ slica gel (100-200 mesh) gravity column with suitable organic solvents. Mass spectra were recorded on Shimadzu GC-MS-QP-2010 model using direct inlet probe technique. 1H NMR, 13C NMR was determined in CDCl3 and DMSO-d6 solution on a Bruker Ac 200 or $400 \mathrm{MHz}$ spectrometer. Satisfactory microanalysis was obtained on Flash EA1112 CHN analyzer. Melting points were determined using digital melting point apparatus and were uncorrected.

\section{Experimental Procedures}

General procedure for the synthesis of Ethyl 4-bromo-1-(4chlorobenzyl)-1H-pyrrole-2-carboxylate (2)

4-Chloro benzyl chloride $(2.10 \mathrm{~mL}, 13.05 \mathrm{mmol})$ was added to a suspension of cesium carbonate $(4.25 \mathrm{~g}, 13.05 \mathrm{mmol})$ and ethyl 4-bromo-1H-pyrrole-2-carboxylate (1) (2.84 g. $13.05 \mathrm{mmol})$ in DMF (35 ml). The reaction mixture was stirred at $60 \mathrm{oC}$ for $6 \mathrm{~h}$ (monitored by TLC). The reaction mixture was poured into water $(25 \mathrm{~mL})$ and extracted with EtOAc $(3 \times 20 \mathrm{~mL})$. The organic layer was washed with saturated aq. $\mathrm{NaHCO} 3(1 \times 20 \mathrm{~mL})$, water $(3 \times 10 \mathrm{~mL})$, brine $(1 \times 10 \mathrm{~mL})$, dried over anhydrous $\mathrm{Na} 2 \mathrm{SO} 4$ and concentrated in vacuum. The product was isolated by silica gel chromatography using Hexane: EtOAc (90:10) to afford $3.78 \mathrm{~g}$ (85\%) of compound (2) as white solid. The product was confirmed by spectral analysis.

Ethyl 4-bromo-1-(4-chlorobenzyl)-1H-pyrrole-2-carboxylate (2): White crystal, yield $85 \%, \mathrm{mp} 232-2340 \mathrm{C}, 1 \mathrm{H}$ NMR (400 MHz, DMSO-d6): $\delta \mathrm{H} 7.5$ (s, 1H, ArH), $7.3(\mathrm{~d}, \mathrm{~J}=8.2 \mathrm{~Hz}, 2 \mathrm{H}, \mathrm{ArH}), 7.1$ (d, J $=8.2 \mathrm{~Hz}, 2 \mathrm{H}, \mathrm{ArH}), 7.0(\mathrm{~s}, 1 \mathrm{H}, \mathrm{ArH}), 4.8(\mathrm{~s}, 2 \mathrm{H}, \mathrm{CH} 2-\mathrm{Ar}), 3.7(\mathrm{q}, 2 \mathrm{H}$, OCH2CH3), 1.4 (t, 3H, CH3CH2); 13C NMR (100 MHz, DMSO-d6): $\delta$ 14.9, 54.2, 59.6, 96.7, 107.2, 120.8, 128.0,130.4, 134.5, 135.1,160.3; EIMS: m/z 341 [M]+; Anal. Calc. for C14H13BrClNO2: C, 49.08\%, H, $3.82 \%, \mathrm{~N}, 4.09 \%$; Found: C, 49.01\%, H, 3.75\%, N, 4.01\%

General procedureeforthesynthesis ofEthyl1-(4-chlorobenzyl)4-phenyl-1H-pyrrole-2-carboxylate (3)
Potassium acetate $(0.914 \mathrm{~g}, 13.98 \mathrm{mmol})$ in water $(3 \mathrm{~mL})$ was added to a solution of 2 (1.91 g, $05.59 \mathrm{mmol})$ and phenylboronic acid (0.82 g, $06.70 \mathrm{mmol})$ in 1,4-Dioxane (25 mL). The reaction mixture was degassed using argon and $\mathrm{Pd}(\mathrm{dppf}) \mathrm{Cl} 2(0.228 \mathrm{~g}$, $0.279 \mathrm{mmol}$ ) was added. The reaction mixture was stirred for 15 $\mathrm{h}$ at $120 \mathrm{oC}$. After completion of the reaction, monitored by TLC, the mixture was cooled to room temperature and the solvent was removed under reduced pressure. Water $(10 \mathrm{~mL})$ was added to the residue and extracted with EtOAc $(3 \times 10 \mathrm{~mL})$. The organic layer was washed with brine $(1 \times 10 \mathrm{~mL})$, dried over anhydrous $\mathrm{Na} 2 \mathrm{SO} 4$ and solvent was evaporated in vacuum. The product was purified by silica gel chromatography using Hexane: EtOAc (5:95) to afford $0.549 \mathrm{~g}(29 \%)$ of compound (3) as white off solid. The product was confirmed by spectral analysis.

Ethyl 1-(4-chlorobenzyl)-4-phenyl-1H-pyrrole-2-carboxylate (3): White crystal, yield $29 \%$ mp 192-194 0C, 1H NMR (400 MHz, DMSO-d6): $\delta \mathrm{H} 7.5$ (s, 1H, ArH), 7.3-7.4 (m, 7H, ArH ), $7.0(\mathrm{~m}, 3 \mathrm{H}$, $\mathrm{ArH}$ ), 4.9 (s, 2H, CH2-Ar), 3.7 (q, 2H, OCH2CH3), 1.3 (t, 3H, CH3CH2); 13C NMR (100 MHz, DMSO-d6): $\delta$ 13.8, 54.9, 60.2, 111.8, 114.7, 121.8, 126.6, 1128.1, 129.0, 129.7, 130.8, 134.9, 136.2, 159.8; EIMS: m/z 339 [M]+; Anal. Calc. for C20H18CINO2: C, 70.69\%, H, 5.34\%, N, 4.12\%; Found: C, 70.60\%, H, 5.28\%, N, 4.04\%.

General procedure for the synthesis of 1-(4-chlorobenzyl)-4phenyl-1H-pyrrole-2-carboxylic acid (4)

Ethyl 1-(4-chlorobenzyl)-4-phenyl-1H-pyrrole-2-carboxylate (3) (0.421 g, $01.24 \mathrm{mmol})$ was dissolved in THF (15 mL) and to that lithium hydroxide $(0.044 \mathrm{~g}, 1.86 \mathrm{mmol})$, dissolved in $4 \mathrm{~mL}$ of water, was added drop wise. The reaction mixture was stirred at room temperature for $16 \mathrm{~h}$. The $\mathrm{pH}$ of the reaction mixture was lowered to 2-3 with $1.0 \mathrm{M}, \mathrm{HCl}$. The mixture was extracted with EtOAc $(3 \times 30 \mathrm{~mL})$. The combined organic layer was dried over anhydrous $\mathrm{Na} 2 \mathrm{SO} 4$ and then concentrated in vacuum. The product was isolated by silica gel chromatography using Hexane: EtOAc (50:50) to obtain $0.367 \mathrm{~g}$ (95\%) of compound (4) as white solid. The product was confirmed by spectral analysis.

1-(4-chlorobenzyl)-4-phenyl-1H-pyrrole-2-carboxylic acid (4): White crystal, yield $95 \%, \mathrm{mp} 223-2250 \mathrm{C}, 1 \mathrm{H}$ NMR (400 MHz, DMSO-d6): $\delta \mathrm{H} 7.7$ (s, 1H, ArH), 7.4-7.6 (m, 7H, ArH), 7.3 (m, 3H, ArH), 4.9 (s, 2H, CH2-Ar); 13C NMR (100 MHz, DMSO-d6): $\delta$ 55.2, 112.1, 114.7, 123.3, 126.9, 128.4, 129.0, 130.4, 131.5, 135.2, 136.4, 162.4; EIMS: m/z 311 [M]+; Anal. Calc. for C18H14CINO2: C, $69.35 \%$, H, 4.53\%, N, 4.49\%; Found: C, 69.27\%, H, 4.48\%, N, 4.41\%. 
General procedure for the synthesis of 1-(4-chlorobenzyl)-4phenyl-1H-pyrrole-2-carboxamides (5a-j)

Compound 4 (0.1 g, $0.320 \mathrm{mmol})$ was dissolved in DMF/ $\mathrm{CH} 2 \mathrm{Cl} 2(15 \mathrm{~mL})$ and cooled to $0 \mathrm{oC}$ to $\mathrm{rt}$. To that EDC.HCl $(0.080 \mathrm{~g}$, $0.42 \mathrm{mmol})$, HOBt (0.056 g, $0.42 \mathrm{mmol})$ and DIPEA/TEA $(0.1 \mathrm{~mL}$, $0.50 \mathrm{mmol}$ ) were added. The reaction mixture was stirred for 0.5 $\mathrm{h}$ and then appropriate amine $(0.352 \mathrm{mmol})$ was added and it was stirred for 20-30 h at room temperature. After completion of the reaction (monitored by TLC), the reaction mixture was diluted with dichloromethane $(20 \mathrm{~mL})$ and washed with saturated aq. $\mathrm{NH} 4 \mathrm{Cl}$ solution $(1 \times 20 \mathrm{~mL})$, water $(1 \times 20 \mathrm{~mL})$ and brine $(1 \times 20 \mathrm{~mL})$. The organic layer was dried over anhydrous $\mathrm{Na} 2 \mathrm{SO} 4$ and concentrated in vacuum. The product was isolated by flash chromatography on silica gel using Hexane: EtOAc (95:5) to afford desired targets (5a-j) (70-94\%) as white to off white solids. In case of DMF, after completion of the reaction, the reaction mixture was poured in to water and the obtained solids were filtered and washed thoroughly with water to afford the desired products. The products were confirmed by 1H NMR, 13C NMR \& EIMS.

1-(4-chlorobenzyl)-N-(4-methoxybenzyl)-4-phenyl-1Hpyrrole-2-carboxamide (5a): Off white crystal, yield $82 \%, \mathrm{mp}$ 253-2550C, 1H NMR (400 MHz, DMSO-d6): $\delta \mathrm{H} 9.3$ (t, 1H, NH), 7.37.5 (m, 9H, ArH), 7.0-7.1 (m, 4H, ArH), 6.8-6.9 (m, 2H, ArH), 4.8 (s, 2H, CH2-Ar), 4.2 (d, 2H, CH2-NH), 3.7 (s, 3H, OCH3); 13C NMR (100 MHz, DMSO-d6): $\delta 42.8,54.7,55.6,113.6,115.9,122.2,126.4$, 127.1, 128.2, 129.4, 130.4, 130.8, 131.4, 135.2, 136.7, 157.2, 162.4; EIMS: m/z 430 [M]+; Anal. Calc. for C26H23ClN2O2: C, 72.47\%, H, 5.38\%, N, 6.50\%; Found: C, 72.38\%, H, 5.26\%, N, 6.40\%.

1-(4-chlorobenzyl)-N-(4-iodobenzyl)-4-phenyl-1H-pyrrole-2carboxamide (5b): Off white crystal, yield $85 \%$, mp 272-2740C, $1 \mathrm{H}$ NMR (400 MHz, DMSO-d6): $\delta \mathrm{H} 9.35$ (t, 1H, NH), 7.65 (d, 2H, ArH), 7.44-7.55 (m, 5H, ArH), 7.39 (s, 1H, ArH), 7.26 (s, 1H, ArH), 7.21 (d, $\mathrm{J}=7.7 \mathrm{~Hz}, 2 \mathrm{H}, \mathrm{ArH}), 7.12$ (d, J = 7.9 Hz, 2H, ArH), $7.00(\mathrm{~d}, 2 \mathrm{H}, \mathrm{ArH})$, 5.52 (s, 2H, CH2-Ar), 4.16 (d, 2H, CH2-NH); 13C NMR (100 MHz, DMSO-d6): $\delta$ 43.2, 54.8, 91.7, 115.6, 121.3, 125.2, 126.8, 128.4, 129.3, 130.6, 131.4, 134.9, 136.7, 138.0, 139.4, 160.4; EIMS: m/z 526 [M]+; Anal. Calc. for C25H20ClIN2O: C, 57.00\%, H, 3.83\%, N, 5.32\%; Found: C, 56.91\%, H, 3.74\%, N, 5.23\%.

1-(4-chlorobenzyl)-N-(1-(2-methoxyphenyl)propan-2-yl)-Nmethyl-4-phenyl-1H-pyrrole-2-carboxamide (5c): Off white crystal, yield $85 \%$, mp 265-2670C, 1H NMR (400 MHz, DMSO-d6): $\delta \mathrm{H} 7.48$ 7.57 (m, 5H, ArH), 7.40 (s, 1H, ArH), 7.32 (s, 1H, ArH), 7.24 (d, 2H, $\mathrm{ArH}), 7.13$ (d, 2H, ArH), 6.80-7.10 (m, 4H, ArH), 5.56 (s, 2H, CH2-Ar), 4.10 (m, 1H, N-CH), 3.68 (s, 3H, OCH3), 3.21 (s, 3H, CH3-N), 2.90 (d, $1 \mathrm{H}, \mathrm{CH}), 2.60$ (d, 1H, CH), 1.12 (d, 3H, CH3-CH); 13C NMR (100 MHz, DMSO-d6): $\delta 17.6,33.2,35.5,54.9,56.3,80.6,111.9,115.8,121.0$, 122.0, 126.2, 127.1, 128.3, 129.4, 130.2, 131.5, 135.7, 136.9, 158.2, 161.6; EIMS: m/z 472 [M]+; Anal. Calc. for C29H29CIN2O2: C, 73.64\%, H, 6.18\%, N, 5.92\%; Found: C, 73.52\%, H, 6.08\%, N, 5.74\%.
1'-(1-(4-chlorobenzyl)-4-phenyl-1H-pyrrole-2-carbonyl) spiro[chromane-2,4'-piperidin]-4-one (5d): Off white crystal, yield

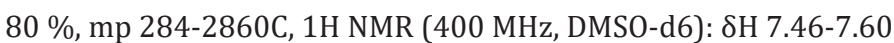
(m, 5H, ArH), 7.41 (s, 1H, ArH), 7.34 (s, 1H, ArH), 7.26 (d, 2H, ArH), 7.10 (d, 2H, ArH), 6.94-7.10 (m, 4H, ArH), 5.51 (s, 2H, CH2-Ar), 3.53.6 (m, 4H, CH2), 2.72 (s, 2H, CH2CO), 1.9-2.1 (m, 4H, CH2); 13C NMR (100 MHz, DMSO-d6): $\delta$ 33.6, 37.4, 43.8, 54.3, 67.6, 113.8, 115.7, 119.5, 121.3, 125.9, 126.8, 127.6, 128.5, 130.2, 130.9, 132.8, 134.6, 135.7, 160.1, 164.6, 191.4EIMS: m/z 510 [M]+; Anal. Calc. for C31H27ClN2O3: C, 72.86\%, H, 5.33\%, N, 5.48\%; Found: C, 72.74\%, H, $5.24 \%$, N, 5.37\%.

7-bromo-1'-(1-(4-chlorobenzyl)-4-phenyl-1H-pyrrole-2carbonyl)spiro[chromane-2,4'-piperidin]-4-one (5e): Off white crystal, yield $85 \%$, mp 291-2930C, 1H NMR (400 MHz, DMSO-d6): $\delta \mathrm{H} 7.8(\mathrm{~d}, 1 \mathrm{H}, \mathrm{ArH}), 7.43-7.50$ (m, 5H, ArH), 7.36 (d, 1H, ArH), 7.32 (s, 1H, ArH), 7.25 (s, 1H, ArH), 7.20 (s, 2H, ArH), 7.15 (d, 2H, ArH), 7.01 (d, 2H, ArH), 5.58 (s, 2H, CH2-Ar), 3.5-3.6 (m, 4H, CH2), 2.70 (s, 2H, CH2CO), 1.9-2.1 (m, 4H, CH2); 13C NMR (100 MHz, DMSO-d6): $\delta$ $34.1,38.2,44.3,55.4,68.5,115.4,116.9,119.8,121.7,122.6,126.3$, 127.2, 128.4, 129.1, 130.2, 131.0, 134.7, 135.6, 158.7, 164.8, 191.2; EIMS: m/z 588 [M]+; Anal. Calc. for C31H26BrClN2O3: C, 63.12\%, H, 4.44\%, N, 4.75\%; Found: C, 63.01\%, H, 4.32\%, N, 4.62\%.

tert-butyl 4-(1-(4-chlorobenzyl)-4-phenyl-1H-pyrrole-2carboxamido)piperidine-1-carboxylate (5f): Off white crystal, yield 79 \%, mp 291-293 0C, 1H NMR (400 MHz, DMSO-d6): $\delta \mathrm{H} 8.2$ (s, 1H, $\mathrm{NH})$, 7.40-7.52 (m, 5H, ArH), $7.34(\mathrm{~s}, 1 \mathrm{H}, \mathrm{ArH}), 7.27$ (s, 1H, ArH), 7.19 (d, 2H, ArH), 7.10 (d, 2H, ArH), 5.58 (s, 2H, CH2-Ar), 3.7 (m, 1H, $\mathrm{CH}$ ), 3.5-3.6 (m, 4H, CH2), 1.8-2.0 (m, 4H, CH2), 1.34 (s, 9H, CH3); 13C NMR (100 MHz, DMSO-d6): $\delta$ 27.6, 28.4, 42.7, 46.3, 54.7, 78.4, 116.3, 121.2, 126.4, 127.8, 129.4, 131.0, 131.6, 134.9, 136.2, 159.2, 161.4; EIMS: m/z 493 [M]+; Anal. Calc. for C28H32CIN3O3: C, $68.07 \%$, H, 6.53\%, N, 8.51\%; Found: C, 67.92\%, H, 6.41\%, N, 8.40\%.

1-(4-chlorobenzyl)-N-cyclohexyl-4-phenyl-1H-pyrrole-2carboxamide (5g): Off white crystal, yield $70 \%, \mathrm{mp} 210-2120 \mathrm{C}, 1 \mathrm{H}$ NMR (400 MHz, DMSO-d6): $\delta \mathrm{H} 8.4$ (b, 1H, NH), 7.38-7.50 (m, 5H, $\operatorname{ArH}), 7.31$ (s, 1H, ArH), 7.24 (s, 1H, ArH), 7.17 (d, 2H, ArH), 7.07 (d, 2H, ArH), 5.53 (s, 2H, CH2-Ar), $3.6(\mathrm{~m}, 1 \mathrm{H}, \mathrm{CH}), 1.5-1.7(\mathrm{~m}, 4 \mathrm{H}$, CH2), 1.43 (m, 2H, CH2), 1.1-1.2 (m, 4H, CH2); 13C NMR (100 MHz, DMSO-d6): $\delta$ 24.2, 25.4, 32.0, 50.6, 53.9, 115.7, 121.4, 125.8, 127.3, 129.1, 129.8, 130.4, 131.6, 135.2, 136.0, 160.6'; EIMS: m/z 392 [M]+; Anal. Calc. for C24H25ClN2O: C, 73.36\%, H, 6.41\%, N, 7.13\%; Found: C, 73.24\%, H, 6.30\%, N, 7.04\%.

(1-(4-chlorobenzyl)-4-phenyl-1H-pyrrol-2-yl) (piperidin-1-yl) methanone (5h): Off white crystal, yield $79 \%$, mp 202-2040C, $1 \mathrm{H}$ NMR (400 MHz, DMSO-d6): $\delta \mathrm{H} \mathrm{7.41-7.56} \mathrm{(m,} \mathrm{5H,} \mathrm{ArH),} 7.36$ (s, 1H, $\operatorname{ArH}), 7.29$ (s, 1H, ArH), 7.23 (d, 2H, ArH), 7.11 (d, 2H, ArH), 5.51 (s, 2H, CH2-Ar), 3.74 (m, 4H, CH2), 1.7 (m, 4H, CH2), 1.6 (m, 2H, CH2); 13C NMR (100 MHz, DMSO-d6): $\delta$ 23.8, 24.9, 46.7, 55.6, 115.8, 120.7, 125.4, 127.1, 128.2, 129.0, 130.3, 131.2, 134.9, 135.8, 164.7; 
EIMS: m/z 378 [M]+; Anal. Calc. for C23H23CIN20: C, 72.91\%, H, $6.12 \%$, N, 7.39\%; Found: C, 72.80\%, H, 6.07\%, N, 7.28\%.

(1-(4-chlorobenzyl)-4-phenyl-1H-pyrrol-2-yl)(pyrrolidin-1-yl) methanone (5i): Off white crystal, yield $74 \%$, mp 196-1980C, 1H NMR (400 MHz, DMSO-d6): $\delta \mathrm{H}$ 7.46-7.59 (m, 5H, ArH), 7.32 (s, 1H, ArH), 7.27 (s, 1H, ArH), 7.21 (d, 2H, ArH), 7.09 (d, 2H, ArH), 5.49 (s, 2H, CH2-Ar), 3.5 (m, 4H, CH2), 1.9 (m, 4H, CH2) ; 13C NMR (100 MHz, DMSO-d6): $\delta 25.4,46.8,55.7,116.3,122.1,127.3,128.1,128.9$, 129.0, 131.1, 131.6, 135.2, 136.3, 165.1; EIMS: m/z 364 [M]+; Anal. Calc. for C22H21ClN2O: C, 72.91\%, H, 6.12\%, N, 7.39\%; Found: C, $72.80 \%, \mathrm{H}, 6.07 \%, \mathrm{~N}, 7.28 \%$.

N'-(1-(4-chlorobenzyl)-4-phenyl-1H-pyrrole-2-carbonyl) isonicotinohydrazide (5j): Off white crystal, yield $72 \%$, mp 2362380C, 1H NMR (400 MHz, DMSO-d6): $\delta \mathrm{H} 11.24$ (b, 1H, NH), 10.72 (b, 1H, NH), 8.84 (d, 2H, ArH), 8.72 (d, 2H, ArH), 7.40-7.58 (m, 5H, ArH), 7.35 (s, 1H, ArH), 7.29 (s, 1H, ArH), 7.23 (d, 2H, ArH), 7.13 (d, 2H, ArH), 5.61 (s, 2H, CH2-Ar); 13C NMR (100 MHz, DMSO-d6): $\delta$ 56.4, 116.3, 121.9, 125.8, 126.4, 128.3, 129.0, 131.2, 131.7, 135.8 136.4, 140.2, 150.4, 161.6, 165.2; EIMS: m/z 430 [M]+; Anal. Calc. for C24H19ClN402: C, 66.90\%, H, 4.44\%, N, 13.0\%; Found: C, $66.78 \%, \mathrm{H}, 4.32 \%, \mathrm{~N}, 12.89 \%$.

\section{Conclusion}

Ten novel 4-phenylpyrrole-2-carboxamide derivatives (5a-j) were prepared from corresponding carboxylic acid and different aromatic or aliphatic or cycloaliphatic amines by employing an efficient amide coupling protocol. The antibacterial and antifungal activity of these compounds (5a-j) was studied against pathogenic Gram-negative bacteria and fungi. The results exhibit that compounds $5 \mathrm{c}$ and $5 \mathrm{e}$ moderate antibacterial activity relative to standard drugs. The compound $5 \mathrm{c}$ displayed moderate antibacterial activity relative to standard against Escherichia coli and Pseudomonas aeruginosa strains with MIC value of $6.05 \mu \mathrm{g} /$ $\mathrm{mL}$. None of these compounds show significant antifungal activity. These results note that presence of lipophilic phenyl group on pyrrole decreases antibacterial activity.

\section{Acknowledgment}

The authors are grateful to the Department of Postgraduate Studies and Research in Chemistry, Dnyanopasak College, Parbhani \& Shri Chhatrapati Shivaji College, Omerga for providing laboratory facilities \& Indian Institute of Chemical Technology, Hyderabad for spectral data.

\section{References}

1. (2009) ECDC EMEA Joint Technical Report, The bacterial challenge: time to react, EMEA576176.

2. Tenover FC, Mc Donald LC (2005) Vancomycin-resistant staphylococci and enterococci: Epidemiology and control. Curr Opin Infect Dis 18(4): 300-305.
3. Pfeltz RF, Wilkinson BJ (2004) The escalating challenge of vancomycin resistance in Staphylococcus aureus. Curr Drug Targets Infect Disord 4(4): 273-294.

4. Roberts MC (2004) Distribution of macrolide, lincosamide, streptogramin, ketolide and oxazolidinone (MLSKO) resistance genes in Gram-negative bacteria. Curr Drug Targets Infect Disord 4(3): 207-215.

5. Dessen A, Di Guilmi AM, Vernet T, Dideberg O (2001) Molecular mechanisms of antibiotic resistance in gram-positive pathogens. Curr Drug Targets Infect Disord 1(1): 63-77.

6. Luca D'Alessandro ED, Giraldi G (2011) A world wide public health problem: The principal re-emerging infectious diseases. Clin Term 162(2): e93-e98.

7. Torres-Viera C, Dembry LM (2004) Approaches to vancomycin-resistant enterococci. Curr Opin Infect Dis 17(6): 541-547.

8. Anstead GM, Owens AD (2004) Recent advances in the treatment of infections due to resistant Staphylococcus aureus. Curr Opin Infect Dis 17(6): 549-555

9. Ford CW, Zurenko GE, Barbachyn MR (2001) The discovery of linezolid, the first oxazolidinone antibacterial agent. Curr Drug Targets Infect Disord 1(2): 181-189.

10. Nina S (2001) Trends in the Epidemiology of Opportunistic Fungal Infections: Predisposing Factors and the Impact of Antimicrobial Use Practices. Clin Infect Dis 33(10): 1692-1696.

11. Fridkin SK, Jarvis WR (1996) Epidemiology of nosocomial fungal infections. Clin Microbiol Rev 9(4): 499-511.

12. Ablordeppey SY, Fan P, Ablordeppey JH, Mardenborough L (1999) Systemic antifungal agents against AIDS-related opportunistic infections: current status and emerging drugs in development. Curr Med Chem 6(12): 1151-1195.

13. Beck-Sague CM, Jarvis WR (1993) Secular trends in the epidemiology of nosocomial fungal infections in the United States, 1980-1990. National Nosocomial Infections Surveillance System. J Infect Dis 167(5): 12471251.

14. Wilkerson WW, Copeland RA, Covington M, Trzaskos JM (1995) J Med Chem 38(20): 3895-3901.

15. Lee H, Lee J, Lee S, Shin Y, Jung W, et al. (2001) A novel class of highly potent, selective, and non-peptidic inhibitor of Ras farnesyltransferase (FTase). Bioorg Med Chem Lett 11(23): 3069-3072.

16. Wurz RP, Charette AB (2005) Doubly Activated Cyclopropanes as Synthetic Precursors for the Preparation of 4-Nitro- and 4-Cyanodihydropyrroles and Pyrroles. Org Lett 7(12): 2313-2316.

17. Piliego C, Holcombe TW, Douglas JD, Woo CH, Beaujuge PM (2010) Synthetic control of structural order in N-alkylthieno[3,4-c]pyrrole-4,6dione-based polymers for efficient solar cells. J Am Chem Soc 132(22): 7595-7597.

18. Katritzky AR, Barczynski P, Musumarra G, Pisano D, Szafran M (1989) Aromaticity as a quantitative concept. 1. A statistical demonstration of the orthogonality of classical and magnetic aromaticity in five- and sixmembered heterocycles. J Am Chem Soc 111(1): 7-15.

19. Etherton BP, Krishnamurti R, Nagy S (1996) US Patent No 5(554): 775.

20. Rigoberto A, Pemites RB (2011) US Patent App. 13/179, 515

21. Kaledkowski A, Trochimczuk AW (2006) Rea Fun Poly 66: 740.

22. Gelling VJ, Wiest MM, Tallman DE, Bierwagen GP, Wallace GG, et al. (2001) Prog Org Coat 43: 149.

23. Rieth RD, Mankad NP, Calimano E, Sadighi JP (2004) Palladium-catalyzed cross-coupling of pyrrole anions with aryl chlorides, bromides, and iodides. Orga Lett 6(22): 3981-3983. 
24. Gribble GW (2003) The diversity of naturally produced organohalogens. Chemosphere 52(2): 289-297.

25. Rane RA, Telvekar VN (2010) Synthesis and evaluation of novel chloropyrrole molecules designed by molecular hybridization of common pharmacophores as potential antimicrobial agents. Bioorg Med Chem Lett 20(19): 5681-5685.

26. Aiello A, Fattorusso E, Menna M, Taglialatela-Scafati O (2008) Wiley-VCH p. 271.

27. Tasdemir D, Topaloglu B, Perozzo R, Brun R, O’Neill R, et al. (2007) Marine natural products from the Turkish sponge Agelas oroides that inhibit the enoyl reductases from Plasmodium falciparum, Mycobacterium tuberculosis and Escherichia coli. Bioorg Med Chem 15(21): 6834-6845.

28. Xiong S, Pang H, Fan J, Ge F, Yang X, et al. (2010) Antitumor activities of a novel indolin-2-ketone compound, Z24: more potent inhibition on bFGF-induced angiogenesis and bcl-2 over-expressing cancer cells. J Pharmacol 159(4): 909-918.

29. Richards J, Ballard T, Huigens R, Melander C (2008) Synthesis and Screening of an Oroidin Library against Pseudomonas aeruginosa Biofilms. Chem Bio Chem 9(8): 1267-1279.

30. Li Y, Zhang P, Ma Q, Song H, Liu Y, et al. (2012) Bioorg Med Chem Lett 22: 6858 .

31. Rane RA, Sahu NU, Shah CP (2012) Synthesis and antibiofilm activity of marine natural product-based 4-thiazolidinones derivatives. Bioorg Med Chem Lett 22(23): 7131-7134.

32. (a) Khalaf AI, Anthony N, Breen D, Donoghue G, Mackayb SP, et al. (2011) Amide isosteres in structure-activity studies of antibacterial minor groove binders. Eur J Med Chem 46(11): 5343- 5355. (b) Anthony NG, Breen D, Donoghue G, Khalaf AI, Mackay SP, et al. (2009) A new synthesis of alkene-containing minor-groove binders and essential hydrogen bonding in binding to DNA and in antibacterial activity. J Org Biomol Chem 7: 1843-1850.

33. Zidar N, Tomasic T, Macut H, Sirc A, Brvar M, et al. (2016) New N-phenyl4,5-dibromopyrrolamides and $\mathrm{N}$-phenylindolamides as ATPase inhibitors of DNA gyrase. Eur J Med Chem 117: 197-211.

34. Hotzel C, Marotto A, Pindur U (2003) New propylamine oligopyrrole carboxamides linked to a heterocyclic or anthraquinone system:

ISSN: 2574-1241

DOI: $10.26717 /$ BJSTR.2020.29.004824

Yogesh D Mane. Biomed J Sci \& Tech Res

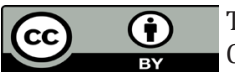

This work is licensed under Creative Commons Attribution 4.0 License

Submission Link: https://biomedres.us/submit-manuscript.php synthesis, DNA binding, topoisomerase I inhibition and cytotoxicity. Eur J Med Chem 38(2): 189-197.

35. Lopez Cara LC M, Camacho E, Dora Carrion M, Tapias V, Gallo MA, et al. (2009) Eur J Med Chem 44: 2655

36. Wang MZ, Xu H, Liu TW, Feng Q Yu SJ, et al. (2011) Design, synthesis and antifungal activities of novel pyrrole alkaloid analogs. Eur J Med Chem 46(5): 1463-1472.

37. Dyson L, Wright AD, Young KA, Sakoff JA, Mc Cluskey A (2014) Synthesis and anticancer activity of focused compound libraries from the natural product lead, oroidin. Bioorg Med Chem 22(5): 1690-1699.

38. Brasca MG, Gnocchi P, Nesi M, Amboldi N, Avanzi N, et al. (2015) Novel pyrrole carboxamide inhibitors of JAK2 as potential treatment of myeloproliferative disorders. Bioorg Med Chem 23(10): 2387-2407.

39. (a) Osman NA, Ligresti A, Klein CD, Allara M, Rabbito A, et al. (2016) Discovery of novel Tetrahydrobenzo[b]thiophene and pyrrole based scaffolds as potent and selective CB2 receptor ligands: The structural elements controlling binding affinity, selectivity and functionality. Eur J Med Chem 122: 619; (b) Ragusa G, Omez-Canas Maria G, Morales P, Hurst DP, Deligia F, et al. (2015) Eur J Med Chem 101: 651.

40. Wang M, Ye C, Liu M, Wu Z, Li L, et al. (2015) Bioorg Med Chem Lett 25: 2782

41. Mane YD, Surwase SM, Biradar DO, Sarnikar YP, Jawle BH, et al. (2017) Design and Synthesis of Diverse Pyrrole-2-carboxamide Derivatives as a Potent Antibacterial Agents. J Heterocyclic Chem 54(5): 2627-2634.

42. (2012) CLSI, Methods for Dilution Antimicrobial Susceptibility Tests for Bacteria that grow Aerobically, Approved Standard, ( $9^{\text {th }}$ edn.), CLSI document M07-A9, Clinical and Laboratory Standards Institute, 950 West Valley Road, Suite 2500, Wayne, Pennsylvania 19087, USA.

43. (2008) CLSI, Reference Method for Broth Dilution Antifungal Susceptibility Testing Filamentous Fungi, Approved Standard, ( $2^{\text {nd }} \mathrm{edn}$.) CLSI document M38-A2, Clinical and Laboratory Standards Institute, 950 West Valley Road, Suite 2500, Wayne, Pennsylvania 19087, USA.

44. (2002) CLSI, Reference Method for Broth Dilution Antifungal Susceptibility Testing of Yeasts, Approved Standard, ( $2^{\text {nd }}$ edn.), NCCLS document M27-A2, Clinical and Laboratory Standards Institute, 940 West Valley Road, Suite 1400, Wayne, Pennsylvania 19087-1898, USA.

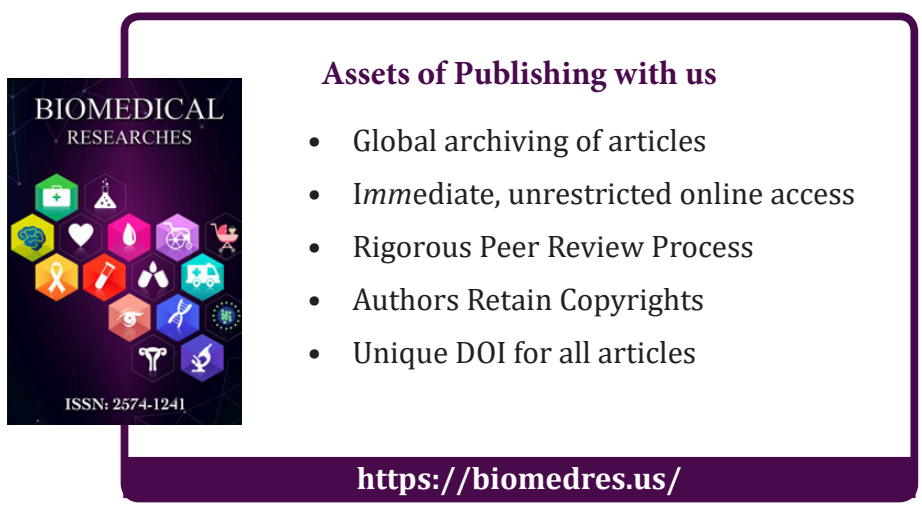

Copyright@ Yogesh D Mane | Biomed J Sci \& Tech Res | BJSTR. MS.ID.004824. 